\title{
The Numerical Solution of the Imaginary-Axis Eliashberg Equations
}

\author{
R. SZCZȨŚNIAK* \\ Institute of Physics, Czȩstochowa University of Technology \\ al. Armii Krajowej 19, 42-200 Czȩstochowa, Poland \\ (Received August 8, 2005; in final form January 9, 2006)
}

\begin{abstract}
In the paper, we solve the imaginary-axis Eliashberg equations. We calculate numerically self-consistently the superconducting order function, the wave function renormalization factor, and the energy shift function as a function of the Matsubara frequency. We consider different values of the average number of the electrons per lattice site. Additionally, we study the temperature dependence of the order function and the wave function renormalization factor. The possible extension of the Eliashberg theory to the case of the high- $T_{\mathrm{C}}$ superconductors was also briefly discussed.
\end{abstract}

PACS numbers: 63.20.Kr, 74.25.Kc

\section{Introduction}

The natural development of the BCS model [1] is the Eliashberg theory $[2,3]$. The Eliashberg model is successfully used to analyze physical properties of the classical phonon-mediated superconductors. The theoretical consideration suggests that this formalism can also be used to analyze thermodynamic properties of the high- $T_{\mathrm{C}}$ superconductors [4].

The starting point of the Eliashberg model is the electron-phonon Hamiltonian (the Fröhlich Hamiltonian [5]) which describes coupling of the electron to an optical phonon mode and the Nambu notation [6]. It is essential that having the Fröhlich Hamiltonian in the Nambu representation, one can obtain the matrix Dyson equation which determines simultaneously normal-state and superconducting properties. In the framework of this formalism one can derive, in a self-consistent manner, the Eliashberg equations. The Eliashberg equations are written on the imaginary frequency axis and create a set of three non-linear integral equations plus the electron number equation. The solutions of this set

*e-mail: szczesni@mim.pcz.czest.pl 
of equations are: the superconducting order function, the wave function renormalization factor, the energy shift function, and the chemical potential. In the Eliashberg scheme the superconducting order function determines the strength of the phonon-mediated potential; the wave function renormalization factor reflects the enhancement of the electron mass due to the interaction effects. The energy shift function characterizes the renormalization of the energy band.

Because the Eliashberg equations have complicated structure, in general, it is impossible to obtain exact analytical results. This means that the analytical method cannot be used to calculate exactly the thermodynamic properties of the phonon-mediated superconductors. The analytical approach can be applied only in qualitative anticipations $[7,8]$.

In the paper, we are interested in the exact solution of the imaginary-axis Eliashberg equations near the transition temperature by using a numerical procedure. We notice that the imaginary-axis Eliashberg equations are amenable to computer analysis. First, because the sums of the Matsubara frequency are discrete. Second, because the order function, wave function renormalization factor, and energy shift function are always real functions.

\section{Model}

\subsection{Eliashberg equations}

In one band calculation the Eliashberg equations form a coupled set of non-linear integral equations which determine the superconducting order function $\varphi_{k}\left(\mathrm{i} \omega_{l}\right)$, the wave function renormalization factor $Z_{k}\left(\mathrm{i} \omega_{l}\right)$, the energy shift function $\chi_{\boldsymbol{k}}\left(\mathrm{i} \omega_{l}\right)$ and chemical potential $\mu$. The symbol $\omega_{l}$ is the Matsubara frequency; $\omega_{l} \equiv(\pi / \beta)(2 l+1) ; \beta$ is the inverse temperature $\beta \equiv\left(k_{\mathrm{B}} T\right)^{-1}$ and $k_{\mathrm{B}}$ is the Boltzmann constant. In our considerations we neglect the momentum dependence of $Z_{\boldsymbol{k}}\left(\mathrm{i} \omega_{l}\right), \chi_{\boldsymbol{k}}\left(\mathrm{i} \omega_{l}\right)$ and $\varphi_{\boldsymbol{k}}\left(\mathrm{i} \omega_{l}\right)$. This approximation is called "the local approximation" and is used when superconductivity is isotropic [3]. To further simplify the notation, we have adopted the shorthand $\varphi\left(\mathrm{i} \omega_{l}\right) \equiv \varphi_{l}, Z\left(\mathrm{i} \omega_{l}\right) \equiv Z_{l}$ and $\chi\left(\mathrm{i} \omega_{l}\right) \equiv \chi_{l}$. Consequently, we can write the Eliashberg equations as follows:

$$
\begin{aligned}
& Z_{l}=1+\frac{1}{\omega_{l} \beta} \sum_{\boldsymbol{k} m} K(l-m) Z_{m} \omega_{m} D_{\boldsymbol{k}}^{-1}(m), \\
& \chi_{l}=-\frac{1}{\beta} \sum_{\boldsymbol{k} m} K(l-m)\left(\varepsilon_{\boldsymbol{k}}+\chi_{m}-\mu\right) D_{\boldsymbol{k}}^{-1}(m), \\
& \varphi_{l}=\frac{1}{\beta} \sum_{\boldsymbol{k} m} K(l-m) \varphi_{m} D_{\boldsymbol{k}}^{-1}(m) .
\end{aligned}
$$

The set of Eqs. (1)-(3) is supplemented with the electron number equation

$$
n=1-\frac{2}{\beta} \sum_{\boldsymbol{k} m}\left(\varepsilon_{\boldsymbol{k}}+\chi_{m}-\mu\right) D_{\boldsymbol{k}}^{-1}(m),
$$

where the symbol $n$ is the average number of the electrons per lattice site (the 
occupation number) and

$$
D_{\boldsymbol{k}}(m) \equiv\left(Z_{m} \omega_{m}\right)^{2}+\left(\varepsilon_{\boldsymbol{k}}+\chi_{m}-\mu\right)^{2}+\varphi_{m}^{2} .
$$

The electronic band structure is modelled by the three-dimensional band with the nearest neighbor hopping integral $t ; \varepsilon_{\boldsymbol{k}}=-2 t\left[\cos \left(a k_{x}\right)+\cos \left(a k_{y}\right)+\right.$ $\left.\cos \left(a k_{z}\right)\right]$. The vector $\boldsymbol{a} \equiv(a, a, a)$ is the basis vector of the simple cubic lattice. In the paper we assume the constant density of states $\rho(0)$ for the electron system. In our case $\rho(0) \equiv 1 /(2 W)$, where $W$ is half of the band width; $W=6 t$. In the numerical calculations we take $t$ as an energy unit. The kernel $K(l-m)$ has the form

$$
K(l-m) \equiv \int_{0}^{+\infty} \mathrm{d} \Omega \frac{2 \alpha^{2} F(\Omega) \Omega}{\left(\omega_{l}-\omega_{m}\right)^{2}+\Omega^{2}},
$$

where $\alpha^{2} F(\Omega)$ is the spectral function. The calculation of the spectral function requires knowledge of the electronic state, the electron density of states, the energy of phonon, and the electron-phonon matrix elements. This calculation is rather complicated. Therefore, we make use of the Kresin simplification and introduce the average phonon frequency $\langle\Omega\rangle[8]$. In our consideration $\langle\Omega\rangle=\omega_{\mathrm{D}} ; \omega_{\mathrm{D}}$ is the Debye frequency. Then we write the kernel $K(l-m)$ as follows:

$$
K(l-m) \simeq \lambda \frac{\nu^{2}}{(l-m)^{2}+\nu^{2}},
$$

where $\nu \equiv \beta \omega_{\mathrm{D}} / 2 \pi$ and $\lambda$ is the electron-phonon coupling function.

In general, the system of Eliashberg equations can be solved numerically. First, we observe that the functions $Z_{l}, \chi_{l}$ and $\varphi_{l}$ are symmetric

$$
Z_{l}=Z_{-(l+1)}, \quad \chi_{l}=\chi_{-(l+1)}, \quad \varphi_{l}=\varphi_{-(l+1)} .
$$

That is why we can rewrite the Eliashberg equations in the appropriate form for numerical analysis

$$
\begin{aligned}
& Z_{l}=1+\frac{1}{\omega_{l} \beta} \sum_{\boldsymbol{k}} \sum_{m=0}^{+\infty}[K(l-m)-K(l+m+1)] Z_{m} \omega_{m} D_{\boldsymbol{k}}^{-1}(m), \\
& \chi_{l}=-\frac{1}{\beta} \sum_{\boldsymbol{k}} \sum_{m=0}^{+\infty}[K(l-m)+K(l+m+1)]\left(\varepsilon_{\boldsymbol{k}}+\chi_{m}-\mu\right) D_{\boldsymbol{k}}^{-1}(m), \\
& \varphi_{l}=\frac{1}{\beta} \sum_{\boldsymbol{k}} \sum_{m=0}^{+\infty}[K(l-m)+K(l+m+1)] \varphi_{m} D_{\boldsymbol{k}}^{-1}(m) .
\end{aligned}
$$

Additionally, we simplify the electron number equation

$$
\begin{aligned}
n \simeq 1 & -\frac{2}{\beta} \sum_{\boldsymbol{k} m} \frac{\varepsilon_{\boldsymbol{k}}-\mu}{\omega_{m}^{2}+\left(\varepsilon_{\boldsymbol{k}}-\mu\right)^{2}} \simeq 1-\rho(0) \int_{-W}^{W} \mathrm{~d} \varepsilon \tanh \left[\frac{\beta(\varepsilon-\mu)}{2}\right] \\
& =1-\frac{2 \rho(0)}{\beta} \ln \left[\frac{\cosh \left(\frac{\beta(W-\mu)}{2}\right)}{\cosh \left(\frac{\beta(W+\mu)}{2}\right)}\right] .
\end{aligned}
$$


We can calculate also the $\boldsymbol{k}$-momentum dependent part of Eqs. (8)-(10). We obtain

$$
\sum_{\boldsymbol{k}} D_{\boldsymbol{k}}^{-1}(m) \simeq \rho(0) \int_{-W}^{W} \mathrm{~d} \varepsilon D_{\varepsilon}^{-1}(m)=D_{1}\left(\omega_{m}, Z_{m}, \chi_{m}, \varphi_{m}, \mu\right)
$$

and

$$
\begin{aligned}
& \sum_{\boldsymbol{k}}\left(\varepsilon_{\boldsymbol{k}}+\chi_{m}-\mu\right) D_{\boldsymbol{k}}^{-1}(m) \simeq \rho(0) \int_{-W}^{W} \mathrm{~d} \varepsilon\left(\varepsilon+\chi_{m}-\mu\right) D_{\varepsilon}^{-1}(m) \\
& \quad=D_{2}\left(\omega_{m}, Z_{m}, \chi_{m}, \varphi_{m}, \mu\right)
\end{aligned}
$$

where

$$
\begin{aligned}
& D_{1}\left(\omega_{m}, Z_{m}, \chi_{m}, \varphi_{m}, \mu\right) \equiv \rho(0) R_{1}\left(\chi_{m}-\mu, \sqrt{\left(Z_{m} \omega_{m}\right)^{2}+\varphi_{m}^{2}}\right), \\
& D_{2}\left(\omega_{m}, Z_{m}, \chi_{m}, \varphi_{m}, \mu\right) \equiv \rho(0) R_{2}\left(\chi_{m}-\mu, \sqrt{\left(Z_{m} \omega_{m}\right)^{2}+\varphi_{m}^{2}}\right) .
\end{aligned}
$$

The function $R_{1}$ and $R_{2}$ are defined by

$$
\begin{aligned}
& R_{1}\left(x_{1}, x_{2}\right) \equiv \frac{1}{x_{2}}\left[\arctan \left(\frac{W+x_{1}}{x_{2}}\right)+\arctan \left(\frac{W-x_{1}}{x_{2}}\right)\right], \\
& R_{2}\left(x_{1}, x_{2}\right) \equiv \frac{1}{2} \ln \left[\frac{\left(W+x_{1}\right)^{2}+x_{2}^{2}}{\left(W-x_{1}\right)^{2}+x_{2}^{2}}\right] .
\end{aligned}
$$

\subsection{Numerical results}

In this part of the paper, we show the exact solution of the Eliashberg equations along the imaginary axis. In Figs. 1-3 we plot the functions $\varphi_{m}, Z_{m}$ and $\chi_{m}$ as functions of the integer number $m$ for different values of the occupation number $n$. We take 1002 values of the number $m$ in the -501 to 500 range. We discuss the properties of $\varphi_{m}, Z_{m}$ and $\chi_{m}$ for positive values of the number $m$, because these

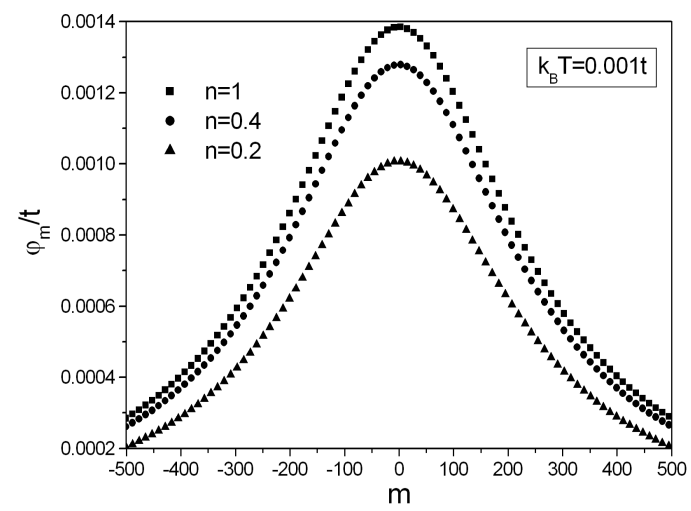

Fig. 1. The order function $\varphi_{m}$ as a function of the number $m$, for different values of the occupation number $n$. We assume $\lambda=2 t, \omega_{\mathrm{D}}=1.5 t$. 


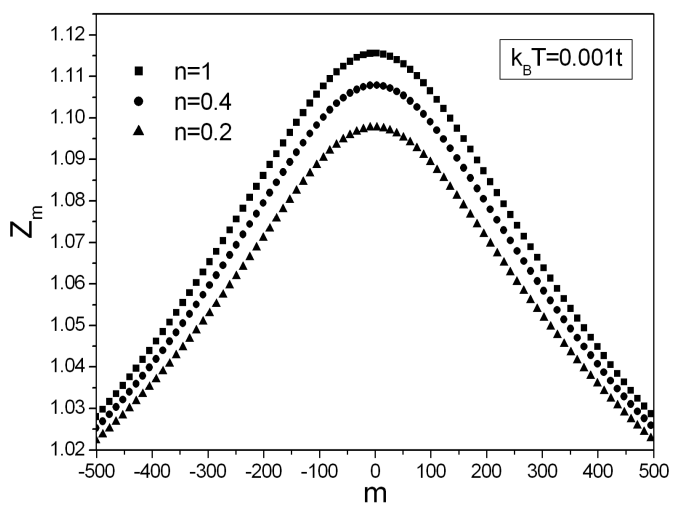

Fig. 2. The wave function renormalization factor $Z_{m}$ as a function of the number $m$, for different values of the occupation number $n$. We assume $\lambda=2 t, \omega_{\mathrm{D}}=1.5 t$.

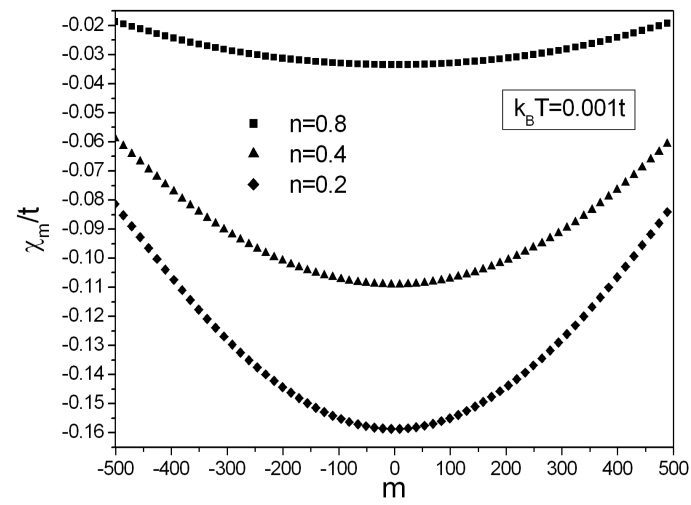

Fig. 3. The energy shift function $\chi_{m}$ as a function of the number $m$, for different values of the occupation number $n$. We assume $\lambda=2 t, \omega_{\mathrm{D}}=1.5 t$.

functions are completely symmetrical in the number $m$. As shown in Fig. 1 and Fig. 2 the functions $\varphi_{m}, Z_{m}$ decrease when the number $m$ increases; what is more, these functions have only positive values. The maximum values of these functions are always for $m=0$. In contrast, the energy shift function $\chi_{m}$ increases when the number $m$ increases, is always negative and has minimum value for $m=0$ (see Fig. 3). Additionally, we show in Figs. 1-3 that the superconducting order function, the wave function renormalization factor, and the energy shift function strongly decrease when the value of the occupation number decreases. We discuss below the exact relationship between $\varphi_{m}, Z_{m}, \chi_{m}$ and $n$.

In Fig. 4 we show selected extreme values of the order function, the wave function renormalization factor, and the energy shift function (we take $\varphi_{m}, Z_{m}$ and $\chi_{m}$ for $m=0$ ) as a function of the occupation number. In Fig. 4 we show also the dependence of the chemical potential on the occupation number. Our 

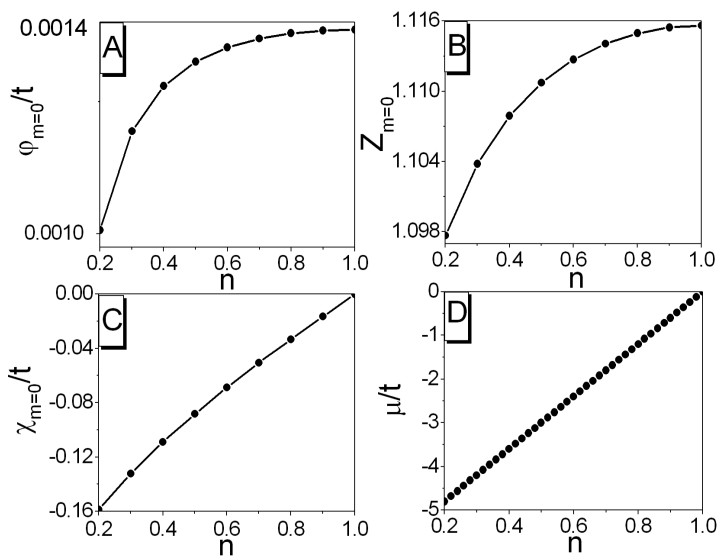

Fig. 4. (A) The order function $\varphi_{m=0}$, (B) the wave function renormalization factor $Z_{m=0},(\mathrm{C})$ the energy shift function $\chi_{m=0}$ and (D) the chemical potential $\mu$ as functions of the occupation number $n$. We assume $k_{\mathrm{B}} T=0.001 t, \lambda=2 t, \omega_{\mathrm{D}}=1.5 t$.

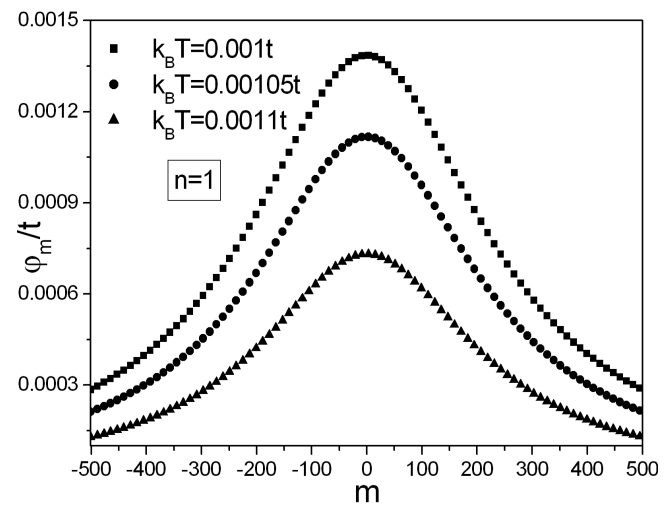

Fig. 5. The order function $\varphi_{m}$ as a function of the number $m$ for different values of the temperature. We assume $\lambda=2 t, \omega_{\mathrm{D}}=1.5 t$.

numerical calculations can prove that the functions $\varphi_{m=0}, Z_{m=0}, \chi_{m=0}$ and $\mu$ decrease when the occupation number decreases from 1 to 0.2 . From the physical point of view this result indicates that the superconducting state is the strongest when the electron band energy is half-filled (the ratio $\varphi_{m} / Z_{m}$ has maximal value for $n=1)$. One can observe also that the known approximate formula for the wave function renormalization factor, $Z \simeq 1+\lambda \rho(0)$, correctly qualitatively reconstructs only the values of $Z_{m}$ for small values of number $m$ and large values of number $n$ (see Fig. 2 and Fig. 4 ). In our case $Z \simeq 1.17$ and $\left[Z_{m=0}\right]_{\max } \simeq 1.12$. For large values of the number $m$ or small values of the occupation number $n, Z$ calculated analytically considerably exceeds the numerical values of $Z_{m}$. Additionally, one can see in Fig. 4 that the values of the energy shift function in comparison with 
the values of the chemical potential are very small. Thus on many occasions, one can neglect the function $\chi_{m}$ in the physical analysis.

In the next step, we assume the half-filled band and consider the order function and the wave function renormalization factor for different values of the temperature close to the transition temperature (in our case the critical temperature is approximately equal to $0.0012 t$ ). We notice also that in case when $n=1$ the energy shift function $\chi_{m}=0$ and $\mu=0$ fulfils the Eliashberg equations. In Fig. 5 we observe the manifestation of the typical behaviour of the order parameter for the function $\varphi_{m}$; when the temperature decreases, the order function $\varphi_{m}$ increases. In contrast to the order function the wave function renormalization factor very weakly depends on the temperature. This results in hinting that the many-body effects connected with the electron-phonon interaction cannot be neglected in the physical analysis. In particular, the renormalization of the electron mass is important. As it is known, this renormalization is directly described by the function $Z_{m}$.

\section{Concluding remarks}

To summarize, we have solved self-consistently the Eliashberg equations for different values of the occupation number. In general, the values of the function $\varphi_{m}, Z_{m}, \chi_{m}$ and $\mu$ decrease when the occupation number decreases. Therefore the $s$-wave superconductivity is the strongest for half-filled electron band energy. Additionally, we have showed that the energy shift function in the whole range of the occupation number has very small value in comparison with the value of the chemical potential. Thus this function can be neglected in the physical analysis. We have also studied the temperature dependence of the order function and the wave function renormalization factor. Considerably important is the fact that the wave function renormalization factor very weakly depends on the temperature, thus the renormalization of the electron mass, connected with the electron-phonon interaction, cannot be neglected in complete physical analysis.

The functions $\varphi_{m}, Z_{m}$ and $\chi_{m}$ are obtained for the minimum temperature equal to $k_{\mathrm{B}} T=0.001 t$. This temperature is near the transition temperature. As our numerical results shows, even for the temperatures close to the transition temperature, the exact calculation of $\varphi_{m}, Z_{m}$ and $\chi_{m}$ requires a consideration of a great number of the Matsubara frequencies. Therefore, the calculation of the order parameter $\Delta_{m} \equiv \varphi_{m} / Z_{m}$ for the zero temperature really on fitting is very complicated.

The results obtained in the paper are correct for the classical phonon-mediated superconductors. For high- $T_{\mathrm{C}}$ superconductors [9] the presented model requires meaningful modification. Among other things, in the high- $T_{\mathrm{C}}$ superconductors the band spectrum is quasi-two-dimensional and strongly anisotropic (the $d$-wave superconductivity plays a dominating role). Therefore, in the simplest case the electronic density of states should not be modelled by the constant but by the 
logarithmic function. In this case, the critical temperature is strongly enhanced from the standard BCS value while the isotope coefficient is small [10].

In the future, in the first step, we will try to analyze the Eliashberg equations in the self-consistent way for the two-dimensional square lattice with the nearest-neighbour hopping integral. We will model the quasi-two-dimensional electron system by the logarithmic singularity in the electronic density of states (the van Hove singularity [10]). We will also consider the role of the van Hove singularity in the presence of the strong Coulomb correlations [11].

\section{Acknowledgments}

The author would like to express the gratitude to Prof. M.B. Zapart and Prof. W. Zapart for creating excellent working conditions and Dr. J. Solecki and MSc. T. Mila for the technical help.

\section{References}

[1] J. Bardeen, L.N. Cooper, J.R. Schrieffer, Phys. Rev. 106, 162 (1957); Phys. Rev. 108, 1175 (1957).

[2] E.M. Eliashberg, Zh. Eksp. Teor. Fiz. 38, 966 (1960) [Sov. Phys. JETP 11, 696 (1960)].

[3] For discussion of the Eliashberg equations [originally formulated by G.M. Eliashberg, Sov. Phys. JETP 11, 696 (1960)] we refer to: P.B. Allen, B. Mitrović, in: Solid State Physics: Advances in Research and Applications, Vol. 37, Eds. H. Ehrenreich, F. Seitz, D. Turnbull, Academic, New York 1982, p. 1; F. Marsiglio, J.P. Carbotte, in: Electron-Phonon Superconductivity, Review for book Handbook on Superconductivity: Conventional and Unconventional Superconductors, Eds. K.H. Bennemann, J.B. Ketterson, Springer, Berlin 2003, p. 233.

[4] M. Mierzejewski, J. Zieliński, Phys. Rev. B 56, 1 (1997).

[5] H. Fröhlich, Phys. Rev. 79, 845 (1950); H. Fröhlich, Proc. R. Soc. A 223, 296 (1954).

[6] Y. Nambu, Phys. Rev. 117, 648 (1960).

[7] W.L. McMillan, Phys. Rev. 167, 331 (1968).

[8] V.Z. Kresin, H. Gutfreund, W.A. Little, Solid State Commun. 51, 339 (1984); V.Z. Kresin, Phys. Lett. A 122, 434 (1987).

[9] J.G. Bednorz, K.A. Müller, Z. Phys. B 64, 189 (1986); J.G. Bednorz, K.A. Müller, Rev. Mod. Phys. 60, 585 (1988).

[10] R. Szczȩśniak, S. Grabiński, Acta Phys. Pol. A 102, 401 (2002); R. Szczȩśniak, M. Mierzejewski, J. Zieliński, P. Entel, Solid State Commun. 117, 369 (2001); R.S. Markiewicz: J. Phys. Chem. Solids 58, 1179 (1997).

[11] J. Hubbard, Proc. R. Soc. Lond. A 276, 238 (1963). 